\title{
ATMOSPHERIC LIDAR NOISE REDUCTION BASED ON ENSEMBLE EMPIRICAL MODE DECOMPOSITION
}

\author{
Jun $\mathrm{LI}^{\mathrm{a}}{ }^{*}$, Wei GONG ${ }^{\mathrm{a}}$, Yingying Ma ${ }^{\mathrm{a}}$ \\ a LIESMARS, Wuhan University, Wuhan 430079, China - larkiner@gmail.com
}

Commission VIII/3

KEY WORDS: LIDAR; Analysis; Algorithms; Atmosphere; Detection

\begin{abstract}
:
As an active remote sensing instrument, lidar provides a high spatial resolution vertical profile of aerosol optical properties. But the effective range and data reliability are often limited by various noises. Performing a proper denoising method will improve the quality of the signals obtained. The denoising method based on ensemble empirical mode decomposition (EEMD) is introduced, but the denoised results are difficult to evaluated. A dual field-of-view lidar for observing atmospheric aerosols is described. The backscattering signals obtained from two channels have different signal-to-noise ratios (SNR). To overcome the drawback of the simulation experiment, the performance of noise reduction can be investigated by comparing the high SNR signal and the denoised low SNR signal. With this approach, some parameters of the denoising method based on EEMD can be determined effectively. The experimental results show that the EEMD-based method with proper parameters can effectively increase the atmospheric lidar observing ability.
\end{abstract}

\section{INTRODUCTION}

\subsection{General Instructions}

Aerosol can directly affect climate change by scattering and absorption of solar and other radiation, and also indirectly affect the radiation by affecting cloud formation. As an active remote sensing instrument, lidar provides a high spatial resolution vertical profile of aerosol optical properties ${ }^{[1]}$. But the effective range and data reliability are often limited by various noises. Unfortunately, the lidar data inversion is sensitive to the lidar data at a far distance, which are under low signal-to-noise ratio conditions. Performing a proper denoising method will improve the quality of the signals obtained.

The measured lidar signal contains the laser backscattering signal from aerosol and various noises. It can be expressed simply as

$$
V_{\text {measured }}(r)=V(r)+N_{b}(r)+N_{e}(r)
$$

where $V_{\text {measured }}(r)=$ signal actually measured $V(r)=$ signal from aerosol backscattering

$N_{b}(r)=$ noise due to background light electronics.

$N_{e}(r)=$ noise due to dark current and read out

$N_{b}$ and $N_{e}$ can be statistically estimated by the signal obtained from a very far distance where the laser backscattering signal is negligible.
The power of the received signal typically falls with an increase in range, but noise is usually considered as Gaussian white noise, which is stable with range. The signal-to-noise ratio (SNR) falls as the range increases, and the solution for the lidar equation becomes unstable and even fails because of the negative value produced by noise. So the signal must be denoised before data retrieval for the aerosol properties.

There are several signal analysis methods widely adopted for the noise reduction in the lidar signal. Most lidar systems employ the multiple pulses averaging to enhance SNR. This method can be considered as a low pass filtering process at the cost of temporal resolution, high frequency backscattering signal is also smoothed. Wavelet analysis is developed rapidly as an effective tool for noise reduction ${ }^{[2]}$. A main drawback of the wavelet analysis is that the basis functions are fixed, and no such a basis function is proposed to correspond with the features of lidar signals currently. The selection of the best basis function is also a hard work.

\section{DENOISING METHOD}

\subsection{Empirical mode decomposition}

Huang et al. introduced the empirical mode decomposition (EMD) for analyzing signals from non-stationary and nonlinear processes in 1998. The EMD method is proved to address completeness, orthogonality, locality, and adaptivity which are necessary to describe non-stationary and non-linear processes. The major advantage of the EMD is posteriori adaptive, because the basis functions are derived from the

* Corresponding author. Jun LI, larkiner@gmail.com 
signal itself. Through a sifting process described by Huang et al., the signal can be decomposed into a series of intrinsic mode functions (IMF) and the residual through the sifting process.

$$
V_{\text {measured }}(r)=\sum_{j=1}^{n} I M F_{j}(r)+R_{n}(r)
$$

Where $\mathrm{IMF}=\mathrm{a}$ series of intrinsic mode functions $\mathrm{R}_{\mathrm{n}}=$ residual

Each IMF satisfies two conditions: the number of extrema and the number of zero crossings must either be equal or differ at most by one, and the mean of the upper and lower envelopes derived from local extrema is zero at any point. This allows for physically meaningful instantaneous frequency and amplitude calculation through the Hilbert transform performed on the IMFs. And any IMF represents a simple oscillatory mode. The low-order IMFs represent high frequency oscillation components, while high-order IMFs represent low frequency oscillation components. The noise and signal are traditionally characterized by high frequency and low frequency, respectively, so they can be distinguished by the empirical mode decomposition method. While the EMD technique has been applied to various fields, although the theoretical base is empirical, some research has shown that the EMD-based signal denoising method is effective in the analysis of a lidar signal .

\subsection{Ensemble empirical mode decomposition}

The empirical mode decomposition results sometimes become invalid because of mode mixing, which is defined as either a single IMF consisting of more oscillatory modes, or an oscillatory mode residing in different IMF.

To overcome the phenomena of mode mixing, the ensemble empirical mode decomposition (EEMD) method has been proposed. This method is a noise assisted data analysis method. The It repeatedly decomposes the signal into IMFs by using the EMD method. During each trial of the decomposition process, white noise is added to the original signal. The final results are obtained as the mean of corresponding IMFs of the

The mode mixing can be effectively eliminated by the EEMD process.

\subsection{EEMD-based denoising method}

The EEMD-based signal denoising method is achieved as follows.

Step 1: Decompose the signal with the EEMD method. At last the original signal is decomposed into a series of IMFs and a trend.

Step 2: Reconstruction. In the time domain, the lower order and higher order IMFs represent the fine scales and coarse scales, respectively. It is assumed that low-order IMFs contain little value of the backscattering signal, and the denoising method is performed by obtaining the residual with the removal of some low-order IMFs.

\section{DATA}

\subsection{The dual field-of-view lidar}

A dual field-of-view lidar (DFL) system was developed by the State Key Laboratory of Information Engineering in Surveying, Mapping and Remote Sensing (LIESMARS), Wuhan University. The lidar has two independent receiving channels to solve the problem of the dynamic range of lidar. The fieldof-view of the near-range and far-range channels is 10 and 1 mrad, respectively. The laser beam fully enters the field-ofview of the near-range and far-range channels from a distance of about $360 \mathrm{~m}$ and $1000 \mathrm{~m}$, respectively.

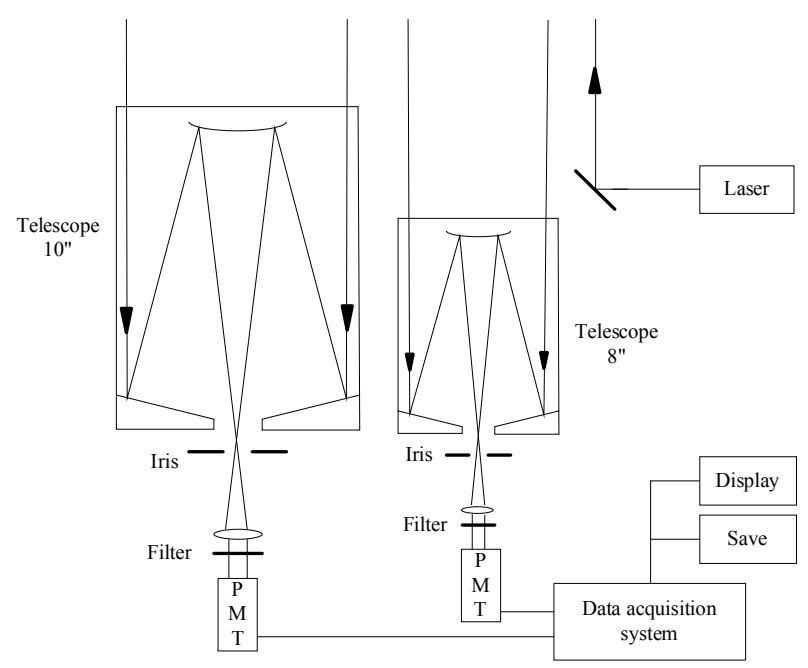

Fig. 1. The schematic diagram of the DFL system.

\section{2 data}

Three pairs data are shown in Fig. 2. They are obtained by the dual field-of-view lidar at 20:10 and 20:12 Aug. 3, and 01:40 Aug. 5.

The comparison of these two simultaneous signals within the range from $1.5 \mathrm{~km}$ to $3.0 \mathrm{~km}$ indicates similar useful signals and obviously different noise. The signals obtained from the two channels are similar because of simultaneous measurements and the same altitude of the atmosphere, and this is evidenced by long-term observing data. The noise intensity of the nearrange channel is higher because of the large field-of-view and low efficiency of the optics and electronics. First, the larger field-of-view means more interference from background light. Second, the signal of the near-range channel is restricted to avoid saturation of the receiver.

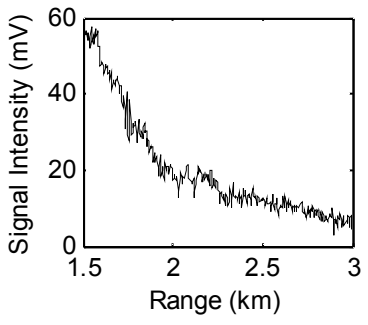

a. Near (20:10)

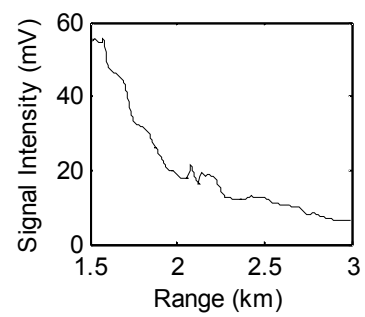

b. Far $(20: 10)$ 


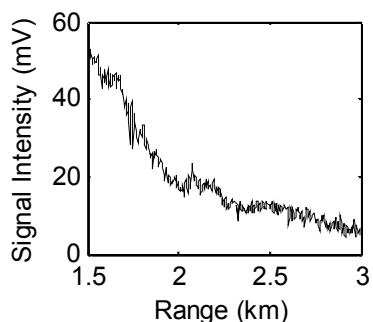

c. Near $(20: 12)$

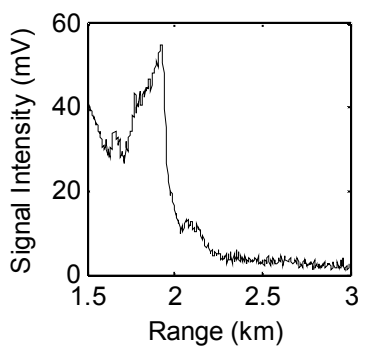

e. Near $(01: 40)$

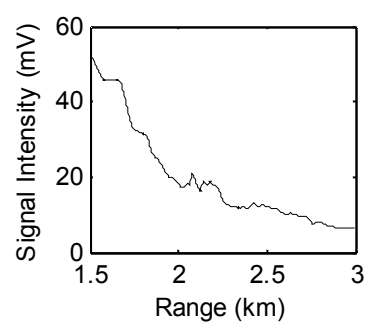

d. Far $(20: 12)$

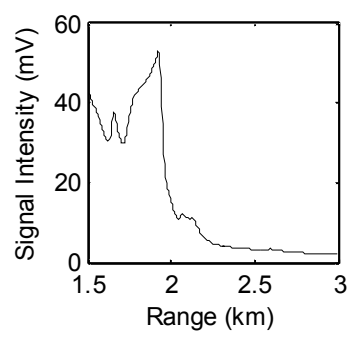

f. $\operatorname{Far}(01: 40)$

Fig. 2. Near and far range signal

\section{RESULT AND DISCUSSION}

The performances of noise reduction are evaluated by overall signal-to-noise ratio (SNR).

$$
S N R=10 * \log \frac{\sum V_{f a r}^{2}(r)}{\sum\left[V_{\text {denoised }}(r)-V_{f a r}(r)\right]^{2}}
$$

where $\mathrm{SNR}=$ overall signal-to-noise ratio

$$
\begin{aligned}
& V_{\text {far }}=\text { far range signal intensity } \\
& V_{\text {denoised }}=\text { denoised near range signal intensity }
\end{aligned}
$$

Three pairs data show in Fig.2 are analyzed. 'Near' is considered as original SNR. 'R2' and 'R3' are EMD-based denoised results by subtracting the first two or three IMFs. 'wavelet' is the wavelet-based denoised result. 'EEMD' is EEMD-based result. Firstly, the SNR of the original signal is relatively low, the SNRs are less than 27 , and denoised results by most method are more than 29. Secondly, SNRs of the 'EEMD' are better than 'EMD' and 'wavelet' method. Finally, the 'EMD' results in Aug. 5 is even less than original data because the aerosols of lower atmosphere changed rapidly in that day. But 'EEMD' method is still effective.

\begin{tabular}{|c|c|c|c|}
\hline & $20: 10$ & $20: 12$ & $01: 40$ \\
& Aug.3 & Aug. 3 & Aug.5 \\
\hline Near & 24.03 & 24.03 & 26.57 \\
R2 & 29.54 & 30.56 & 26.18 \\
R3 & 29.65 & 29.44 & 8.59 \\
wavelet & 29.26 & 30.13 & 30.14 \\
EEMD & 30.22 & 31.73 & 30.29 \\
\hline
\end{tabular}

Table 1. SNR of different denoising algorithms

\section{CONCLUSIONS}

The primary result shows that the EEMD-based method can effectively increase the lidar observing ability. The result is promising and further work is required to evaluated the performance of noise reduction in different lidar system and atmospheric environment.

\section{REFERENCES}

N. E. Huang, Z. Wu, 2008. A review on Hilbert-Huang transform: Method and its applications to geophysical studies, Rev. Geophys. 46, RG2006 .

N. E. Huang, Z. Shen, S. R. Long, M. C. Wu, H. H. Shih, Q. Zheng, N. C. Yen, C. C. Tung, H. H. Liu, 1998. The empirical mode decomposition and the Hilbert spectrum for nonlinear and non-stationary time series analysis, Proc. Roy. Soc. Lond. A 454, 903.

W. Gong, J. Li, F. Y. Mao, J. Y. Zhang, 2011. Comparison of simultaneous signals obtained from a dual-field-of-view lidar and its application to noise reduction based on empirical mode decomposition, Chin. Opt. Lett. 9, 5,050101.

Z. Wu and N. E. Huang, 2009. Ensemble empirical mode decomposition: a noise assisted data analysis method. Advance in adaptive data analysis. 1,1 .

S. H. Wu, Z. S. Liu, B. Y. Liu, 2006, Enhancement of lidar backscatters signal-to-noise ratio using empirical mode decomposition method, Opt. Commun. 267, 137.

J. Harms, 1979, Lidar return signals for coaxial and noncoaxial systems with central obstruction, Appl. Opt. 18, 1559 A. O. Boudraa, J. C. Cexus, Z. Saidi, 2004, EMD-based signal noise reduction, Int. J. Signal Process. 1, 33.

\section{ACKNOWLEDGEMENTS}

This work was supported by 973 Program (2009CB723905, 2011CB707106), the NSFC (10978003, 41127901). 